\title{
Luteal Start of Stimulation in a Case of Expected Poor Response with the Successful Outcome: A Case Report
}

\author{
Shruti Gupta
}

\begin{abstract}
Purpose: Poor responder is a quasi-cluster of patients whose management has confounded clinicians. Luteal phase stimulation as a solution is proposed on a new principle of follicular development.

Case description: Mrs. X, a 34-year-old woman with an anti-Mullerian hormone (AMH) of $0.86 \mathrm{ng} / \mathrm{mL}$ and a history of failed in vitro fertilization (IVF) with the recovery of one egg was stimulated in the luteal phase. The patient's ovarian stimulation was done with menopur 375 IU, cetrotide $0.25 \mathrm{mg}$ was added after 8 days. The stimulation lasted for 15 days.

Results: There were seven oocytes recovered, two blastocysts were formed and transferred in a freeze-thaw cycle. This resulted in a live-born preterm fetus at 27 weeks in view of bleeding placenta previa.

Conclusion: Luteal phase stimulation can be another reasonable solution in the long list of stimulation regimens for patients who are poor responders.

Keywords: Luteal start, Poor responder, Random start.

International Journal of Infertility and Fetal Medicine (2020): 10.5005/jp-journals-10016-1206
\end{abstract}

\section{INTRODUCTION}

Poor responders have often confounded in vitro fertilization (IVF) specialists around the world. Much of the research is directed to the finding of reasonable solutions in this group of patients. The lack of a universal definition has induced a considerable degree of heterogeneity to research on stimulation protocols in poor responders.

Random start of stimulation was started for women with malignancy and disorders, where limited time is available to the patient before beginning cytotoxic and gonadotoxic treatment. This has given us information that luteal stimulation even when longer may be associated with a higher number of eggs retrieved.

Here, we are reporting a case where this approach was used in an established poor responder patient with endometriosis.

\section{Case Description}

Patient $X$, a 34-year-old woman with 4 years of infertility was operated on laparoscopically 1 year ago where grade IV endometriosis was diagnosed, and cystectomy was performed bilaterally. Postoperatively, she underwent one cycle of superovulation and intrauterine insemination (IUI), which was unsuccessful. Thereafter, a cycle of IVF was performed elsewhere. The stimulation was an antagonist cycle, which started with 225 IU of recombinant follicle-stimulating hormone (FSH) (Gonal F, Merck Serono, Italy), and 150 IU luteinizing hormone (LH) (Luveris, Merck Serono, Italy). The cycle resulted in the retrieval of one egg, which did not fertilize.

The patient presented with the above history and on the investigation, her anti-Mullerian hormone (AMH) was $0.86 \mathrm{ng} /$ $\mathrm{mL}$ and antral follicle count (AFC) was $6(2+4)$. The patient was planned for a luteal start of stimulation. The experimental nature of the procedure was explained to her.
Indira IVF Hospitals, Bengaluru, Karnataka, India

Corresponding Author: Shruti Gupta, Indira IVF Hospitals, Bengaluru, Karnataka, India, Phone: +91 9990703946, e-mail: shrutisgrh@gmail. com

How to cite this article: Gupta S. Luteal Start of Stimulation in a Case of Expected Poor Response with the Successful Outcome: A Case Report. Int J Infertil Fetal Med 2020;11(2):54-56.

Source of support: Nil

Conflict of interest: None

\section{Stimulation}

The patient was called on day $10 / 26$ day of her cycle. A follicle of $14 \mathrm{~mm}$ was seen in her right ovary. On further follow-up on day 14 at the follicle size of $19 \mathrm{~mm}$, ovulation was triggered with inj. Triptorelin (Decapeptyl, Ferring, Switzerland) 0.2 mg. Ovulation was confirmed on day 16 on ultrasound (USG) and progesterone levels in the blood. The AFC on day 16/D1 of stimulation was 6 (2 +4 ). Stimulation was started with urinary highly purified human menopausal gonadotropin, u-hMG 375 IU (Menopur, Ferring, Switzerland). When the lead follicle was $12 \mathrm{~mm}$ in diameter and cetrorelix acetate was added (cetrotide, Merck Serono, Italy). Egg retrieval was planned at 36 hours of inj. triptorelin $0.2 \mathrm{mg}$. She had seven oocytes retrieved, three metaphase II (MII), two metaphase I (MI), and the rest were germinal vesicle (GV). The MII and MI oocytes (after in vitro maturation) were injected with sperms. Intracytoplasmic sperm injection (ICSI) was performed in view of previous fertilization failure. There were three 2PN embryos at 24 hours and on day 3, (1) 8 cell grade I + (2) 8 cell grade II $(20 \%$ fragmentation). The embryos were frozen for transfer at a later date because of asynchrony between the embryo and the endometrium in luteal phase stimulation.

The embryo transfer was done in a down-regulated, programd hormone replacement cycle in view of her adenomyosis and 
endometriosis. The patient was administered a depot injection of triptorelin (Decapeptyl, Ferring, Switzerland) $3.75 \mathrm{mg}$ and after 3 weeks estrogen was started (Estrabet, Abbott India Ltd.). She was started on injectable progesterone intramuscularly (Gestone, Ferring, Switzerland) $100 \mathrm{mg}$ at an endometrial thickness of 10 $\mathrm{mm}$. The embryos on thawing were cultured to blastocysts. At 118 hours, two embryos were transferred (3.1.1 and 2.1.2, Istanbul consensus). ${ }^{1}$ Her b-hcg was $846 \mathrm{IU} / \mathrm{mL}$ and clinical pregnancy was confirmed at 6 weeks.

The patient developed some bleeding at 20 weeks when the lower segment placenta was made note of. The patient developed leaking at 26 weeks with heavy bleeding at 27 weeks when a lower segment cesarean was performed in view of antepartum hemorrhage and major placenta previa. The baby is a healthy 1,000 $\mathrm{g}$ preterm and is doing well.

\section{Discussion}

In the present patient, the luteal phase was induced by decapeptyl trigger and confirmed by progesterone levels before starting the stimulation. We were successful in the luteal phase to obtain good quality mature oocytes in this patient with documented poor response. Researches have demonstrated the luteal phase to be equally productive in patients with neoplasia and normal responders. ${ }^{2}$ The usage of the luteal phase has never been demonstrated in the poor responder. Dual stimulation (shanghai protocol) has been studied in poor responders and a yield of a higher number of oocytes in the second stimulation has been achieved. ${ }^{3}$ Kuang et al. obtained $3.5 \pm 3.2$ oocytes in the luteal phase as compared to $1.7 \pm 1.0$ in the follicular phase. This formed the background for the work done in the present patient where stimulation began in the second half of the cycle.

According to a new theory on follicular development, there are multiple cohorts developing follicles in a menstrual cycle., ${ }^{4,5}$ This purports that superovulation akin to IVF cycles can effectively begin at any day of the cycle. In relation to this theory random start, late follicular start and luteal start regimes have been proposed. Most of the evidence in poor responders comes from "duostim" (Shanghai protocol). ${ }^{6}$ Accordingly, most of the researchers have obtained the same number of oocytes and blastocysts for transfer in follicular and luteal phase stimulation. ${ }^{2,7}$

Other studies have found no difference in the outcome of stimulation in different parts of the menstrual cycle in terms of eggs retrieved and embryos formed. ${ }^{2,8,9}$ In our experience, we found that the stimulation was prolonged (15 days) and required a higher dose of gonadotropin but ended with the recovery of a significantly higher number of oocytes (Table 1). This was also the observation of Cakmak et al. that the total dose is higher in the late follicular phase and luteal phase. ${ }^{10}$

More recently, researchers in Greece accumulated oocytes from unstimulated cycles in a total of three follicular, luteal, and follicular phases. One hundred and fifty-three women were included and there was no statistically observed difference in the metrics of the outcome either in terms of eggs retrieved or embryos formed. ${ }^{11}$

More evidence is required to improve the performance of luteal phase stimulation, e.g., it has been suggested that antagonists may not be required for controlling $\mathrm{LH}$ surge because of the presence of progesterone in the hormonal milieu. ${ }^{12}$ Kuang et al. studied 242 women in the luteal phase and none of them had a spontaneous LH surge without the antagonist. Follicle-stimulating hormone,
Table 1: Luteal phase follicular stimulation in Mrs. X

\begin{tabular}{|c|c|c|c|}
\hline $\begin{array}{l}\text { Date/day of stimula- } \\
\text { tion }\end{array}$ & Injection & Right ovary & Left ovary \\
\hline 12/10/2017/day 1 & Menopur 375 & $3(3)$ & $4(3)$ \\
\hline 13/10/2017/day 2 & Menopur 375 & & \\
\hline 14/10/2017/day 3 & Menopur 375 & & \\
\hline 15/10/2017/day 4 & Menopur 375 & $7,6,5,5$ & $6(3)$ \\
\hline 16/10/2017/day 5 & Menopur 375 & & \\
\hline $17 / 10 / 2017 /$ day 6 & Menopur 375 & & \\
\hline 18/10/2017/day 7 & Menopur 375 & $7(4)$ & $7,6(3)$ \\
\hline 19/10/2017/day 8 & Menopur 450 & & \\
\hline 20/10/2017/day 9 & $\begin{array}{l}\text { Menopur } 450 / \\
\text { cetrotide } 0.25\end{array}$ & $12,11,9,8$ & 9,8 \\
\hline 21/10/2017/day 10 & $\begin{array}{l}\text { Menopur } 450 / \\
\text { cetrotide } 0.25\end{array}$ & & \\
\hline 22/10/2017/day 11 & $\begin{array}{l}\text { Menopur } 450 / \\
\text { cetrotide } 0.25\end{array}$ & & \\
\hline 23/10/2017/day 12 & $\begin{array}{l}\text { Menopur } 450 / \\
\text { cetrotide } 0.25\end{array}$ & & \\
\hline 24/10/2017/day 13 & $\begin{array}{l}\text { Menopur } 450 / \\
\text { cetrotide } 0.25\end{array}$ & $17,15(2), 13$ & $14(2), 10(3)$ \\
\hline 25/10/2017/day 14 & $\begin{array}{l}\text { Menopur } 450 / \\
\text { cetrotide } 0.25\end{array}$ & & \\
\hline 26/10/2017/day 15 & $\begin{array}{l}\text { Menopur } 450 / \\
\text { cetrotide } 0.25\end{array}$ & $19,18,17,15$ & 15,14 \\
\hline
\end{tabular}

(Cimadomo D 2018)

LH, E2 (estradiol), and P4 (progesterone) were studied, though it is unclear how often the same were monitored. ${ }^{13}$

\section{References}

1. Alpha Scientists in Reproductive Medicine and ESHRE Special Interest Group of Embryology. The Istanbul consensus workshop on embryo assessment: proceedings of an expert meeting. Hum Reprod 2011. $1-14$.

2. Wang N, Wang Y, Chen Q, et al. Luteal-phase ovarian stimulation vs conventional ovarian stimulation in patients with normal ovarian reserve treated for IVF: A large retrospective cohort study. Clin Endocrinol (Oxf) 2016;84(5):720. DOI: 10.1111/cen.12983.

3. Kuang $Y, C$ hen $Q$, Hong $Q$, et al. Double stimulations during the follicular and luteal phases of poor responders in IVF/ICSI programmes (Shanghai protocol). Reproduct Bio Med Online 2014;29(6):684-691. DOI: 10.1016/j.rbmo.2014.08.009.

4. Mikolajczyk RT, Frank-Herrmann P, Freundl G, et al. More than one fertile ovulation per cycle? Fertil Steril 2004;81(3):728-729. DOI: 10.1016/j.fertnstert.2003.11.016.

5. Baerwald AR, Adams GP, Pierson RA. Characterization of ovarian follicular wave dynamics in women. Biol Reprod 2003;69(3):10231031. DOI: $10.1095 /$ biolreprod.103.017772.

6. Moffat R, Pirtea P, Gayet V, et al. Dual ovarian stimulation is a new viable option for enhancing the oocyte yield when the time for assisted reproductive technnology is limited. Reprod Biomed Online 2014;29(6):659-661. DOI: 10.1016/j.rbmo.2014.08.010.

7. Kansal Kalra S, Ratcliffe S, Gracia CR, et al. Randomized controlled pilot trial of luteal phase recombinant FSH stimulation in poor responders. Reprod Biomed Online 2008;17(6):745-750. DOI: 10.1016/ S1472-6483(10)60400-2.

8. Qin N, Chen Q, Hong Q, et al. Flexibility in starting ovarian stimulation at different phases of the menstrual cycle for treatment of infertile women with the use of in vitro fertilization or intracytoplasmic 
sperm injection. Fertil Steril 2016;106(2):334-341. DOI: 10.1016/j. fertnstert.2016.04.006.

9. Ubaldi F, Capalbo A, Vaiarelli A, et al. Follicular versus luteal phase ovarian stimulation during the same menstrual cycle (DuoStim) in a reduced ovarian reserve population results in a similar euploid blastocyst formation rate: New insight in ovarian reserve exploitation. Fertil Steril 2016;105(6):1488-1495. DOI: 10.1016/j. fertnstert.2016.03.002.

10. Cakmak H, Katz A, Cedars MI, et al. Effective method for emergency fertility preservation: random-start controlled ovarian stimulation. Fertil Steril 2013;100(6):1673-1680. DOI: 10.1016/j. fertnstert.2013.07.1992.

11. Sfakianoudis K, Simopoulou M, Maziotis E, et al. Evaluation of the second follicular wave phenomenon in natural cycle assisted reproduction: a key option for poor responders through luteal phase oocyte retrieval. Medicina (Kaunas) 2019;55(3):68. DOI: 10.3390/ medicina55030068.

12. Zhu $\mathrm{X}, \mathrm{Ye} \mathrm{H}, \mathrm{Fu} \mathrm{Y}$. Use of Utrogestan during controlled ovarian hyperstimulation in normally ovulating women undergoing in vitro fertilization or intracytoplasmic sperm injection treatments in combination with a "freeze all" strategy: a randomized controlled dose-finding study of $100 \mathrm{mg}$ versus $200 \mathrm{mg}$. Fertil Steril 2017;107(2):379-386. DOI: 10.1016/j.fertnstert.2016. 10.030 .

13. Kuang $Y$, Hong $Q$, Chen $Q$, et al. Luteal-phase ovarian stimulation is feasible for producing competent oocytes in women undergoing in vitro fertilization/intracytoplasmic sperm injection treatment, with optimal pregnancy outcomes in frozen-thawed embryo transfer cycles. Fertil Steril 2014;101(1):105-111. DOI: 10.1016/j. fertnstert.2013.09.007. 